\title{
Pancreatic Serous Cystadenocarcinoma: A Case Report and Review of the Literature
}

\author{
Jonathan C. King • Tina T. Ng • Stephen C. White • \\ Galen Cortina • Howard A. Reber • O. Joe Hines
}

Received: 16 April 2009/Accepted: 28 April 2009/Published online: 21 May 2009

(C) 2009 The Author(s). This article is published with open access at Springerlink.com

\begin{abstract}
Background Serous cystic neoplasms of the pancreas are benign lesions with little chance for malignant degeneration. We report a case of malignant serous cystadenocarcinoma of the pancreas and review the literature.

Methods Structured review of the literature was performed using PubMed and MEDLINE searches, and cases of serous cystadenocarcinoma of the pancreas were compiled.

Results A 70-year-old man diagnosed with a serous cystadenoma was managed expectantly until he became symptomatic, and studies revealed an increase in the size of the lesion as well as duodenal invasion. The patient underwent a pancreaticoduodenectomy, and histopathological examination revealed a locally invasive cystadenocarcinoma without metastatic disease. Seven years later, the patient remains disease-free. Review of the literature identified 25 cases of serous cystadenocarcinoma published to date. The mean age at diagnosis is $68 \pm 2$ years (range, 52 to 81 ), and women are affected more commonly $(2: 1)$.

Conclusions We conclude that there is a small but finite risk of malignancy for serous cystic neoplasms of the pancreas. The clinician should bear this in mind when faced with decisions regarding patient management. Prognosis is excellent with multiple reports of long-term survival even in the face of metastatic disease.
\end{abstract}

Keywords Pancreas · Oncology · Serous cystic lesion .

Serous cystadenoma $\cdot$ Serous cystadenocarcinoma

\section{Introduction}

Malignant cystic neoplasms are rare entities that account for only $1 \%$ of all pancreatic tumors. ${ }^{1}$ Serous and mucinous cystic neoplasms are tumors of the exocrine pancreas with

J. C. King • T. T. Ng • S. C. White $\cdot$ H. A. Reber • O. J. Hines $(\triangle)$ Department of Surgery,

David Geffen School of Medicine at UCLA, Box 956904, 72-170 CHS,

Los Angeles, CA 90095-6904, USA

e-mail: joehines@mednet.ucla.edu

G. Cortina

Department of Pathology,

David Geffen School of Medicine at UCLA,

Los Angeles, CA, USA different biological behaviors. Mucinous cystic tumors are typically slow-growing but carry a significant potential for malignancy, and thus, resection is often indicated. ${ }^{2,3}$ In contrast, serous cystadenomas are considered benign tumors with almost no malignant potential. They are often observed with serial imaging or managed expectantly. ${ }^{4}$ In the absence of symptoms, surgery is not usually recommended.

The first case of a pancreatic serous cystadenocarcinoma was reported by George et al. in 1989. The authors described the malignant characteristics of a serous cystic tumor of the pancreas with invasion into the spleen, stomach, and liver. The patient expired intra-operatively due to hemorrhage. ${ }^{5}$ Subsequently, additional reports have documented similar findings of serous cystic neoplasms with malignant behavior. The histological characteristics of serous cystadenocarcinoma are indistinguishable from its benign counterpart, making the presence of invasion the sole distinguishing characteristic between the two. ${ }^{5}$ In this report, we present a case of serous cystadenocarcinoma with duodenal, vascular, and neural invasion. We also 
review the literature and discuss the current diagnostic techniques and principles of management.

\section{Materials and Methods}

A systematic review of the literature was performed utilizing PubMed and MEDLINE searches. Articles were identified using the search terms: pancreas and serous cystadenocarcinoma. Nineteen articles were included in the analysis. Data are presented as mean \pm standard error of the mean.

\section{Results}

A 70-year-old man presented with upper gastrointestinal (GI) bleeding and abdominal pain. There was a duodenal ulcer with no evidence of malignancy on esophagogastroduodenoscopy (EGD), and an abdominal computed tomography $(\mathrm{CT})$ scan revealed a $5.7-\mathrm{cm}$ cystic mass in the head of the pancreas which was diagnosed by core needle biopsy as a serous cystadenoma. The patient was treated for presumed duodenal ulcer disease leading to resolution of symptoms and scheduled for observation of his pancreatic mass.

Three months later, the patient returned with recurrent coffee-ground emesis and abdominal pain. CT scan showed enlargement of the pancreatic mass to $6.5 \times 8 \mathrm{~cm}$ and central dystrophic calcifications with new pancreatic and biliary ductal dilatation (Fig. 1). Repeat EGD identified a bleeding duodenal ulceration, and biopsies were consistent with a "benign" serous cystadenoma.

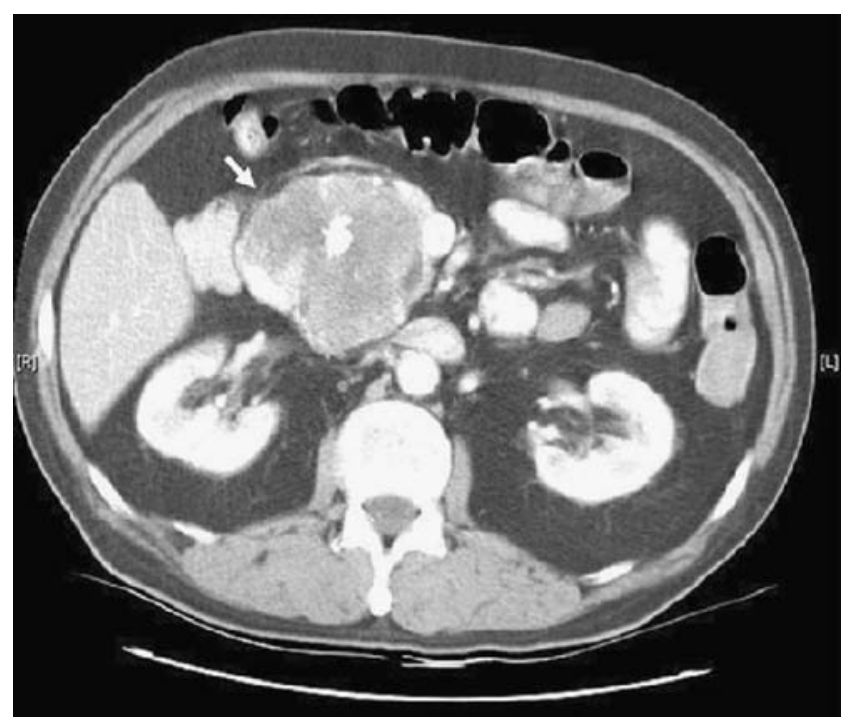

Fig. 1 Contrast-enhanced CT scan demonstrating a large mass measuring $6.5 \times 8 \mathrm{~cm}$ in the head of the pancreas (arrow) with central dystrophic calcifications.
Upon surgical consultation, an elective pancreaticoduodenectomy was planned due to the increased size of the tumor, recurrent bleeding, and erosion into the duodenum. Laparotomy revealed a large mass in the head of the pancreas with no evidence of gross metastatic disease or invasion of the mesenteric vessels. The patient's postoperative course was complicated by delayed gastric emptying requiring temporary gastrostomy and feeding jejunostomy tubes.

On gross examination, the mass measured $9 \times 8 \times 6 \mathrm{~cm}$, and there was marked, aggressive invasion of the duodenum beyond the level of the muscularis propria (Fig. 2). Histology demonstrated microcysts lined by clear cells without mucinous cytoplasm (Fig. 3). Microscopic vascular and perineural invasion were also seen, further distinguishing this lesion from a benign serous cystadenoma (Figs. 4 and 5). All resection margins and 17 lymph nodes were uninvolved.

Immunochemical stains for keratin AE1/3, 7, and 19 and CAM 5.2 were positive. Stained samples of the mass also showed weak immunoreactivity for carcinoembryonic antigen (CEA). Keratin 20 staining was negative. DNA content analysis by flow cytometry demonstrated no evidence of aneuploidy.

The final diagnosis was serous cystadenocarcinoma with duodenal, vascular, and neural invasion. At last follow-up 7 years post-operatively, the patient is doing well without clinical or radiographic evidence of recurrent disease.

Literature review yielded 25 reports of serous cystadenocarcinoma (Table 1). ${ }^{5-23}$ The average age at presentation is $68 \pm 2$ years, and $60 \%$ of patients affected are female ( $28 \%$ male; in $12 \%$ of cases, sex was not reported). Presenting complaints included abdominal pain (24\%), upper GI bleeding (12\%), weight loss $(8 \%)$, palpable mass $(8 \%)$, jaundice or abnormal serum liver enzymes $(8 \%)$, and nonspecific abdominal complaints (8\%).

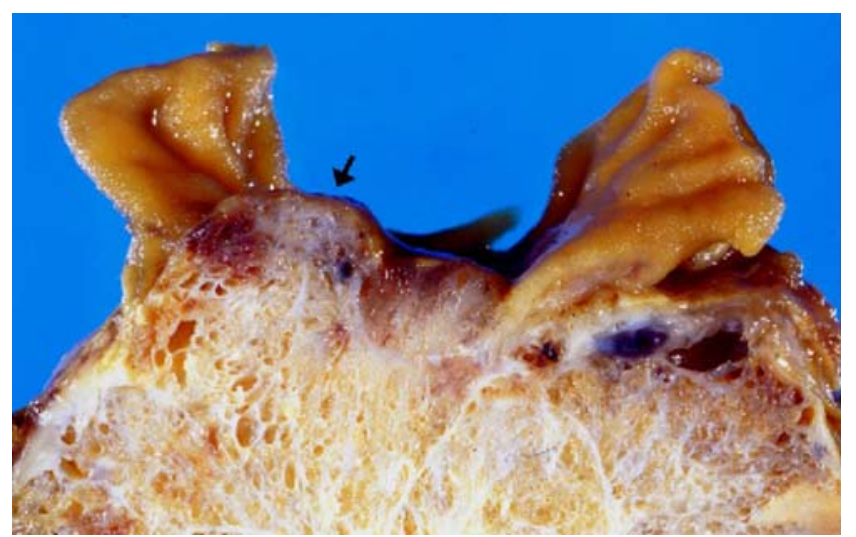

Fig. 2 Gross appearance of the tumor. Note invasion of the duodenum through the muscularis propria and submucosa with ulceration of the overlying mucosa (arrow). 


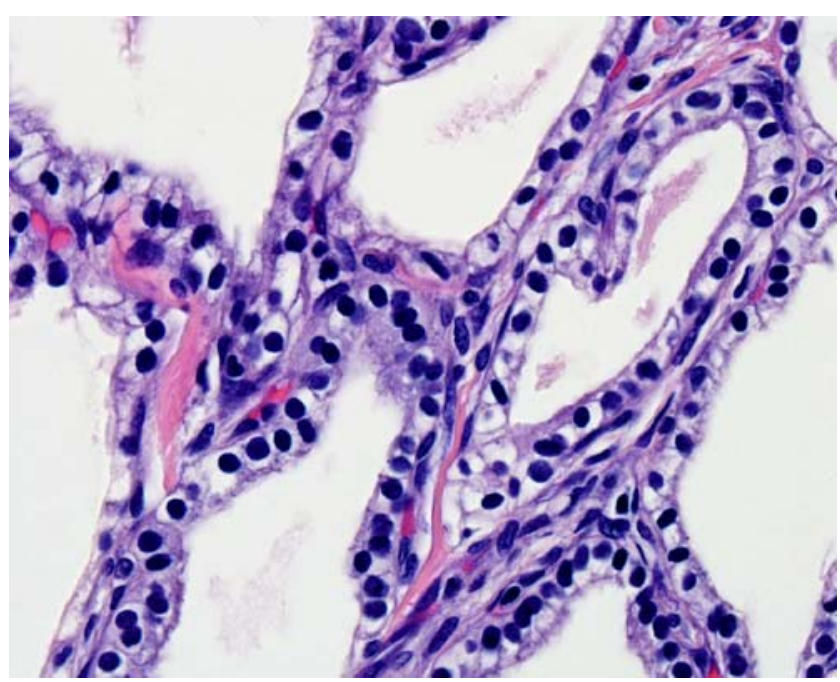

Fig. 3 Microscopic appearance of the tumor. The tumor is composed of multiple cysts lined by cuboidal cells with clear cytoplasm.

The mean diameter of serous cystadenocarcinoma was $10 \pm 1 \mathrm{~cm}$ (range, $2.5-19 \mathrm{~cm}$ ). Lesions exhibit both of the hallmarks of malignancy: local invasiveness and distant metastasis with most tumors associated with local invasion of the spleen (8\%), small intestine (4\%), stomach $(4 \%)$, adrenal gland (4\%), or microscopic invasion of vascular and neural tissues. Synchronous or metachronous liver metastases were frequently noted (36\%), along with metastasis to regional lymph nodes $(12 \%)$, bone marrow (4\%), and lung (4\%). Mean survival was $36 \pm 11$ months (range, $<30$ days to 120 months) among cases with followup $(n=11)$, and ten $(91 \%)$ of these patients were still alive when reports were published including seven (64\%) patients with metastatic disease.

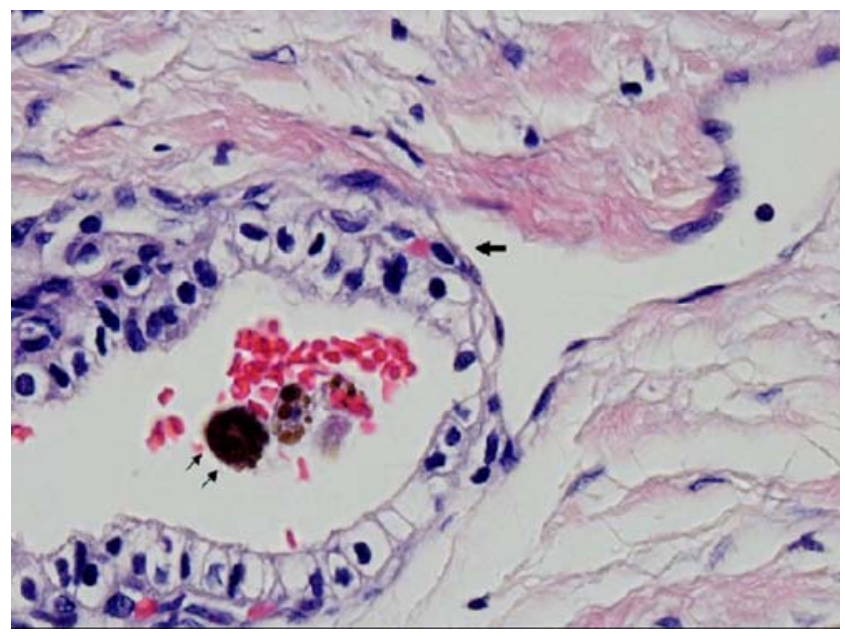

Fig. 4 Vascular invasion. Tumor erodes through the lumen of the vessel (arrow). Red blood cells and hemosiderin (double arrow) are present.

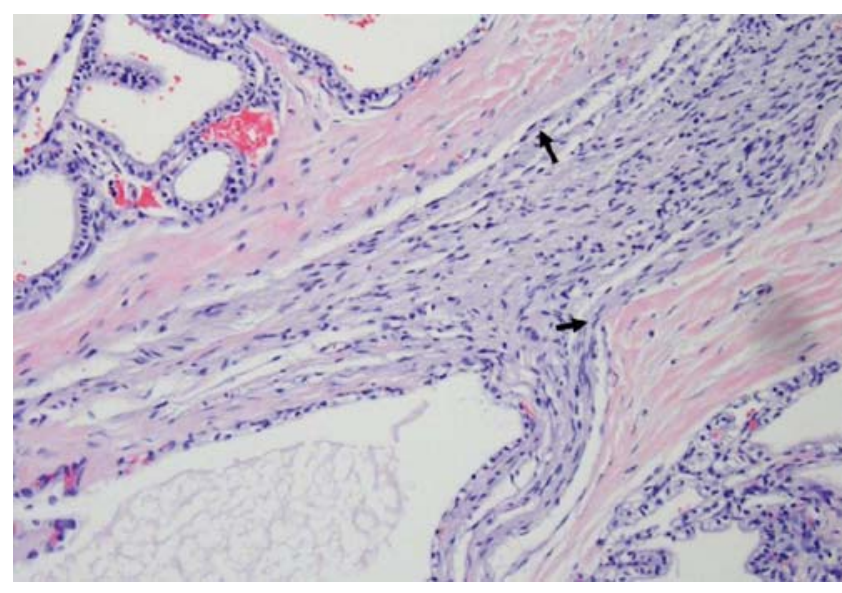

Fig. 5 Neural invasion. Tumor cells are noted within the nerve sheath (arrows).

\section{Discussion}

The preoperative differentiation between a benign serous cystadenoma and malignant serous cystadenocarcinoma remains difficult. Indeed, the correct diagnosis of serous cystadenocarcinoma was not made pre-operatively in any of the cases, including the current one. ${ }^{5-23}$ The benign and malignant variants appear identical histologically, with the only distinguishing feature being gross or microscopic evidence of invasiveness. Thus, the utility of cytology or histology obtained from core needle biopsy is limited. ${ }^{18}$

The current practice for management of serous cystadenomas of the pancreas is to observe asymptomatic lesions thereby avoiding the potential morbidity and mortality associated with a major operation. ${ }^{13,14,18,24,25}$ We agree with this conservative approach. Nevertheless, clinicians should be aware of the possibility for malignant transformation in serous cystic neoplasms and should maintain an index of suspicion when certain clues appear. These include the onset of new symptoms, worsening of symptoms, or rapid enlargement of the mass. In these cases, resection may be indicated, despite the lack of objective evidence for malignancy obtained from preoperative imaging, endoscopy, and biopsies.

In the current case, a serous cystadenocarcinoma was diagnosed without evidence of distant metastasis but with extensive tumor invasion into surrounding structures, both grossly and microscopically. To our knowledge, this is the first example of serous cystadenocarcinoma with extensive duodenal, vascular, and neural invasion but no distant metastases.

\section{Conclusion}

Our case report is illustrative of the management strategy for serous cystic lesions of the pancreas despite the 


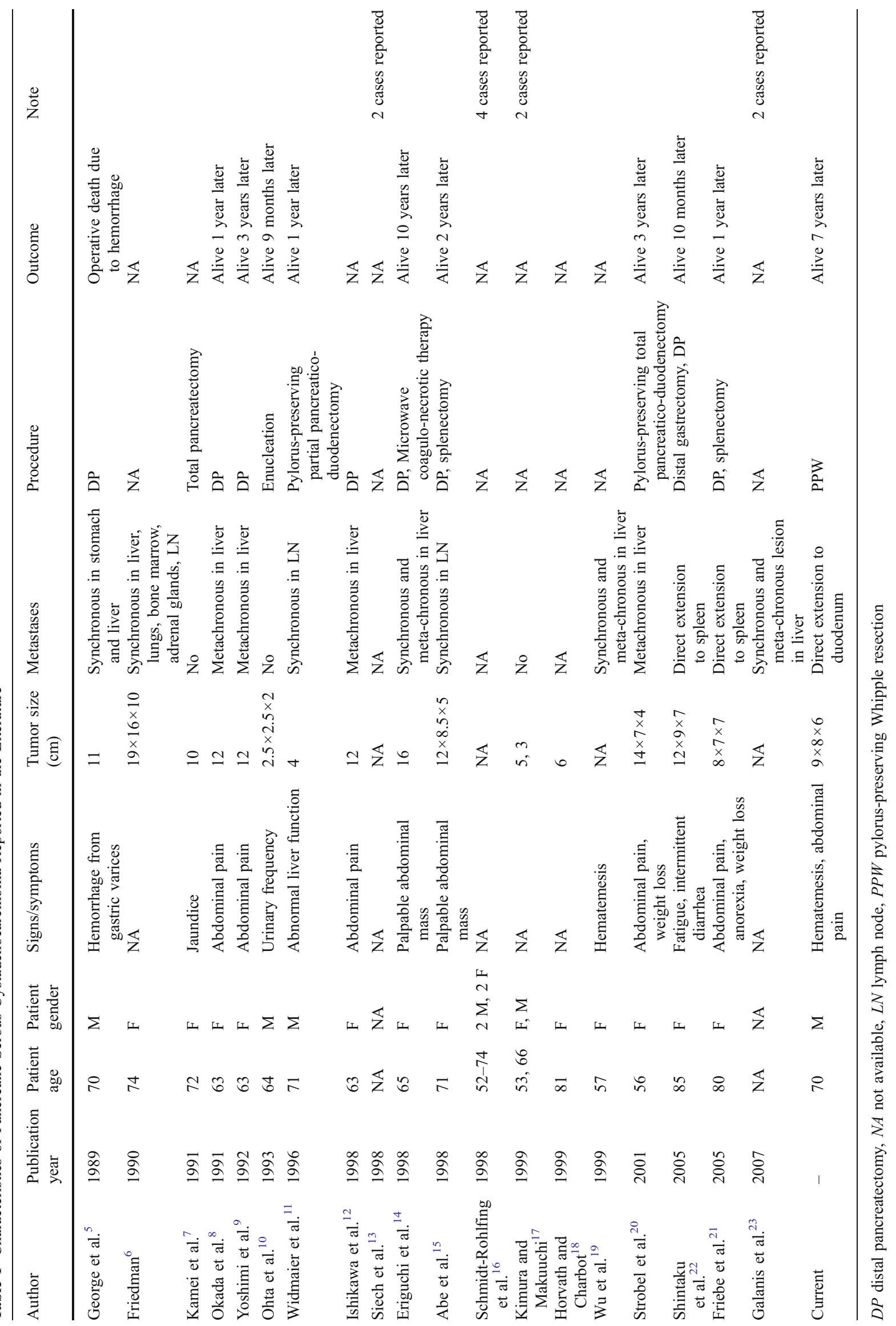


presence of an initially unrecognized malignancy: the progression of symptoms and increase in size of the mass triggered curative resection. The excellent prognosis associated with serous cystadenocarcinoma justifies an aggressive approach to surgical resection, even in older patients. This is especially so since major pancreatic resections are now done with very low mortality and morbidity rates in major centers around the world. ${ }^{26}$

Open Access This article is distributed under the terms of the Creative Commons Attribution Noncommercial License which permits any noncommercial use, distribution, and reproduction in any medium, provided the original author(s) and source are credited.

\section{References}

1. Becker WF, Welsh A, Pratt HS. Cystadenoma and cystadenocarcinoma of the pancreas. Ann Surg 1965;161:845-860. doi:10.1097/00000658-196506000-00005.

2. Klöppel G, Solcia E, Longnecker DS, Capella C, Sobin LH. Histological typing of tumors of the exocrine pancreas, 2nd edition. WHO international histological classification of tumors. Berlin: Springer, 1996.

3. Compagno J, Oertel JE. Mucinous cystic neoplasms of the pancreas with overt and latent malignancy (cystadenocarcinoma and cystadenoma): A clinicopathological study of 41 cases. Am J Clin Pathol 1978;69:573-580.

4. Compagno J, Oertel JE. Microcystic adenomas of the pancreas (glycogen-rich cystadenomas). Am J Clin Pathol 1978;69:289298.

5. George DH, Murphy F, Michalski R, Ulmer BG. Serous cystadenocarcinoma of the pancreas: A new entity? Am J Surg Pathol 1989;13:61-66. doi:10.1097/00000478-19890100000009 .

6. Friedmann HD. Nonmucinous, glycogen-poor cystadenocarcinoma of the pancreas. Arch Pathol Lab Med 1990;114:888-891.

7. Kamei K, Funabiki T, Ochiai M, Amano H, Kasahara M, Sakamoto T. Multifocal pancreatic serous cystadenoma with atypical cells and focal perineural invasion. Int $\mathrm{J}$ Pancreatol 1991;10:161-172.

8. Okada T, Nonami T, Miwa T, Yamada F, Ando K, Tatematsu A, Sugie S, Kondo T. Hepatic metastasis of serous cystadenocarcinoma resected 4 years after operation of the primary tumor: A case report. Jpn J Gastroenterol 1991;88:2719-2723.

9. Yoshimi N, Sugie S, Tanaka T, Aijin W, Bunai Y, Tatematsu O, Mori H. A rare case of cystadenocarcinoma of the pancreas. Cancer 1992;69:2449-2453. doi:10.1002/1097-0142(19920515) 69:10<2449::AID-CNCR2820691011>3.0.CO;2-9.

10. Ohta T, Nagakawa T, Itho H, Fonseca L, Miyazaki I, Terada T. A case of serous cystadenoma of the pancreas with focal malignant changes. Int J Pancreatol 1993;14:283-289.
11. Widmaier U, Mattfeldt T, Siech M. Serous cystadenocarcinoma of the pancreas. Int J Pancreatol 1996;20:135-139.

12. Ishikawa $\mathrm{T}$, Nakao A, Nomoto $\mathrm{S}$. Immunohistochemical and molecular biological studies of serous cystadenoma of the pancreas. Pancreas 1998;16:40-44. doi:10.1097/00006676199801000-00007.

13. Siech M, Tripp K, Schmidt-Rohlfing B, Mattfeldt T, Widmaier U, Gansauge F, Görich J, Beger HG. Cystic tumors of the pancreas: Diagnostic accuracy, pathologic observations and surgical consequences. Langenbecks Arch Surg 1998;383:56-61. doi:10.1007/ s004230050092.

14. Eriguchi N, Aoyagi S, Nakayama T, Hara M, Miyazaki T, Kutami $\mathrm{R}$, Jimi A. Serous cystadenocarcinoma of the pancreas with liver metastases. J Hepatobiliary Pancreat Surg 1998;5:467-470. doi:10.1007/s005340050075.

15. Abe H, Kubota K, Mori M, Miki K, Minagawa M, Noie T, Kimura W, Makuuchi M. Serous cystadenoma of the pancreas with invasive growth: Benign or malignant? Am J Gastroenterol 1998;93:1963-1966. doi:10.1111/j.1572-0241.1998.00556.x.

16. Schmidt-Rohlfing B, Siech M, Mattfeldt T, Schoenberg MH, Beger HG. Cystic neoplasms of the pancreas: Surgical treatment and outcome. Z Gastroenterol 1998;36:939-945.

17. Kimura W, Makuuchi M. Operative indications for cystic lesions of the pancreas with malignant potential: Our experience. Hepatogastroenterology 1999;46:483-491.

18. Horvath K, Charbot JA. An aggressive resectional approach to cystic neoplasms of the pancreas. Am J Surg 1999;178:262-274. doi:10.1016/S0002-9610(99)00186-5.

19. Wu CM, Fisherman EK, Hruban RK. Serous cystic neoplasm involving the pancreas and liver: An unusual clinical entity. Abdom Imaging 1999;24:75-77. doi:10.1007/s002619900445.

20. Strobel O, Z'graggen K, Schmitz-Winnenthal FH, Friess H, Kappeler A, Zimmermann A, Uhl W, Büchler MW. Risk of malignancy in serous cystic neoplasms of the pancreas. Digestion 2003;68:24-33. doi:10.1159/000073222.

21. Friebe V, Keck T, Mattern D, Schmitt-Graeff A, Werner M, Mikami Y, Adam U, Hopt UT. Serous cystadenocarcinoma of the pancreas: Management of a rare entity. Pancreas 2005;31:182187. doi:10.1097/01.mpa.0000167001.89018.3c.

22. Shintaku M, Arimoto A, Sakita N. Serous cystadenocarcinoma of the pancreas. Pathol Int 2005;55:436-439. doi:10.1111/j.14401827.2005.01850.x.

23. Galanis C, Zamani A, Cameron JL, Campbell KA, Lillemoe KD, Caparrelli D, Chang D, Hruban RH, Yeo CJ. Resected serous cystic neoplasms of the pancreas: A review of 158 patients with recommendations for treatment. J Gastrointest Surg 2007;11:820 826. doi:10.1007/s11605-007-0157-4.

24. Omeroglu A, Paner GP, Ciesla MC, Hartman G. Serous microcystic adenoma of the pancreas. Arch Pathol Lab Med 2001;125:1613-1614.

25. Tseng JF, Warshaw AL, Sahani DV, Lauwers GY, Rattner DW, Fernandez-del Castillo C. Serous cystadenoma of the pancreas: Tumor growth rates and recommendations for treatment. Ann Surg 2005;242:413-421.

26. Kazanjian KK, Hines OJ, Duffy JP, Yoon DY, Cortina G, Reber HA. Improved survival following pancreaticoduodenectomy to treat adenocarcinoma of the pancreas: The influence of operative blood loss. Arch Surg 2008;14:1166-1171. 